\title{
CREENCIAS SOBRE EL EMBARAZO, PARTO Y PUERPERIO EN LA MUJER MAPUCHE: CONVERSACIONES PRIVADAS
}

\author{
PREGNANCY, DELIVERY, AND POST PARTUM BELIEFS AMONG MAPUCHE \\ WOMEN: PRIVATE CONVERSATIONS
}

\author{
Ana M. Alarcón ${ }^{1}$ y Yolanda Nahuelcheo $S^{2}$
}

\begin{abstract}
Este trabajo describe los hallazgos de un estudio sobre las percepciones y creencias acerca del embarazo, parto y puerperio en mujeres mapuche de la Región de la Araucanía, Chile.

Los resultados demuestran la presencia de un complejo sistema de cuidado cultural para la mujer mapuche en esta etapa de la vida. Un conjunto de normas sociales y culturales definen lo que es y no es permitido para la embarazada y puérpera, entre las que destacan rigurosas pautas de alimentación, prohibición de participar en ciertos eventos sociales y prevenir el encuentro con espíritus o seres de la cultura mapuche que puedan dañar a la embarazada o al feto. El modelo de cuidado de salud reproductiva relatado por las mujeres combina elementos físicos, naturales, medioambientales y espirituales que obedecen tanto a los paradigmas de la medicina popular como de la medicina mapuche. Por ejemplo, la mantención del equilibrio corporal entre frío y calor, así como el cumplimiento de normas de la cultura mapuche, moldean las conductas de salud de la mujer. El conocimiento de estos cuidados culturales podría contribuir al desarrollo de un enfoque intercultural en el programa de salud de la mujer en nuestro país.
\end{abstract}

Palabras claves: mujer mapuche, embarazo, parto, puerperio.

This study describes the perceptions and beliefs about pregnancy, delivery, and the post partum period among Mapuche women in the Araucania region.

The results demonstrate the existence of a complex system of cultural care for the Mapuche woman in this stage of her life. Social and cultural rules delimit what is allowed during pregnancy and the postpartum period. Among these we found rigorous feeding rules, the prohibition of participation in certain social events, and the prevention of contact with Mapuche spirits that could harm the pregnant women or her child. The cultural pattern of care combines physical, environmental, and spiritual elements of both conventional and Mapuche medicine. For example, maintaining a balance between cold and heat, as well as obeying Mapuche cultural rules, shape women's health behaviours. Knowledge of these cultural patterns of care could contribute to the development of intercultural activities within the woman's health program in our country.

Key words: Mapuche women, pregnancy, childbirth, post partum.

El embarazo, parto y puerperio en la mujer mapuche constituyen procesos de gran importancia social y cultural para esta sociedad, sin embargo han sido escasamente estudiados desde la antropología. Todas las sociedades (modernas o tradicionales) moldean el comportamiento reproductivo de sus miembros (Browner y Sargent 1996). Los patrones culturales de reproducción incluyen las creencias y prácticas relacionadas con la menstruación, embarazo, parto, puerperio, definiciones de roles masculino o femenino, reglas de matrimonio. La antropología médica, y en particular la antropología de la reproducción, plantea que el estudio de estos patrones proporciona un poderoso lente a través del cual es posible observar procesos sociales y culturales más amplios. Por ejemplo, la forma en que una sociedad organiza la conducta reproductiva de sus miembros refleja el conjunto de valores y principios que rigen la propia estructura de la sociedad. Este modo de concebir la reproducción humana implica un análisis que asocia la conducta biológica a procesos socioculturales de perpetuación de los sistemas sociales (Harris y Young 1991). En esta misma línea los enfoques feministas agregan que el concepto de reproducción humana implica el estudio de las estructuras políticas e ideológicas que moldean la producción humana (Beneria y Roldan 1987; Stephen 1991).

En la cultura mapuche los modelos reproductivos han experimentado importantes transformaciones producto del contacto con la sociedad chilena. Por ejemplo, las políticas de un Estado monocultural,

1 Dpto. Salud Pública-CIGES, Facultad de Medicina, Universidad de La Frontera. Manuel Montt 112, Temuco, Chile. amalarc@ufro.cl

2 Secretaria Regional Ministerial de Salud IX Región. Aldunate 70, Temuco, Chile. yolanda.nahuelcheo@ redsalud.gov.cl

Recibido: marzo 2006. Aceptado: agosto 2008. 
que han penalizado prácticas y agentes terapéuticos tradicionales y la creación de programas de salud de carácter nacional para captar a la embarazada y niño mapuche, han sido exitosas desde un análisis estrictamente biomédico (Andrade 1992). Sin embargo, estos logros se han acompañado de profundos procesos de cambio cultural en la familia mapuche, tanto en su modo de concebir el embarazo, parto y puerperio, como en la expresión de las prácticas culturales asociadas a estos fenómenos. Frente a la tensión que involucra reconocer los beneficios biológicos de la medicina del Estado, y dejar las prácticas culturales ancestrales, la mujer ha tenido que reconstruir un mundo de significaciones para adaptar su conducta cultural a un espacio ajeno -el hospital, el consultorio, la posta rural-, a nuevos personajes que controlan su cuerpo y privacidad -la matrona, el médico, la enfermera-, y a nuevas tecnologías y procedimientos -la sala de parto, el quirófano y el instrumental obstétrico-. En este proceso forzado y hegemónico de adaptación, es que la pérdida paulatina del conocimiento como patrimonio cultural alcanza un nivel crítico para la sociedad dominada. La conversión conceptual es hacia una hibridización del conocimiento tradicional indígena, es un cambio en esencia epistemológico y de las lógicas de las explicaciones sobre los fenómenos (García Canclini 2005; Wolf 1999). En consecuencia, hoy poco queda de aquellos relatos historiográficos realizados, entre otros, por Gusinde (1917), Guevara (1912), Faron (1964) y Hillger (1957), de fenómenos asociados a la reproducción mapuche que demostraban un cuerpo simbólicocultural propio de una sociedad "intacta".

En la actualidad, las tensiones culturales derivadas de los procesos de tradición y modernidad, en el contexto de la reproducción humana, confluyen en un nuevo fenómeno sociopolítico y cultural, la interculturalidad en salud, concebida como la posibilidad de un diálogo democrático, o trato igualitario, entre los sistemas de salud tradicional-indígena y la biomedicina (Alarcón et al. 2004). Es precisamente en este nuevo espacio de interacciones sociales y culturales, que la sociedad mapuche podría preservar, manifestar y revitalizar la cultura propia. La Región de la Araucanía ha sido pionera en el intento de incorporar la cosmovisión de salud y enfermedad de la sociedad mapuche en las prácticas de atención de salud.

Este estudio se inscribe en el marco de la antropología reproductiva y tiene el propósito de develar las dimensiones culturales asociadas al embarazo, parto y puerperio en la mujer mapuche; dimensiones que podrían integrarse al programa de la mujer del Ministerio de Salud como una forma de profundizar el diálogo intercultural antes mencionado.

\section{Material y Método}

El presente estudio constituye parte de un trabajo etnográfico realizado durante ocho años en seis comunidades mapuches de la comuna de Nueva Imperial en la IX Región y cuyo propósito fue reconocer los procesos culturales de salud y enfermedad de la sociedad mapuche en interacción con el sistema occidental de salud.

Las participantes en este estudio fueron 12 mujeres, quienes accedieron voluntariamente a relatar sus experiencias y conocimientos acerca del embarazo, parto y puerperio en tanto madres, abuelas y esposas. Asimismo, producto de la extensión del trabajo y la permanencia periódica del equipo investigador en terreno, se tuvo la oportunidad de observar en el grupo de estudio el curso de embarazos y nacimientos, acompañar a algunas de las embarazadas a sus controles de salud, llevarlas al hospital para tener sus hijos, apoyarlas durante los distintos períodos del parto y participar en las actividades cotidianas con sus recién nacidos.

Las características de las mujeres participantes fueron: edad promedio de 25 años (rango 17 a 65), diez eran casadas, dos solteras y tenían en promedio tres hijos. Seis mujeres eran abuelas, quienes tuvieron parto domiciliario y habían ayudado a otras mujeres en el trabajo de parto; las restantes seis mujeres habían tenido sus partos en el hospital. Además de las actividades desarrolladas en el hogar, las mujeres tejían a telar y salían ocasionalmente a vender flores y hortalizas a la ciudad de Temuco. Cuatro mujeres del estudio eran, también, dirigentes de su comunidad, y estaban vinculadas a un programa de formación-acción de promotores de salud rural auspiciado por un organismo no gubernamental de la Región. Todas las participantes eran bilingües (mapudungun-español) y tenían una escolaridad promedio de seis años.

Los datos fueron obtenidos a través de entrevistas en profundidad realizadas en los hogares de las mujeres, por medio de sesiones grupales de debate organizadas en sus casas y de observación participante de las embarazadas y puérperas del 
grupo que pasaron por este proceso durante el desarrollo de la investigación.

Las sesiones grupales y la observación participante contribuyeron respectivamente a profundizar el análisis, a obtener una opinión colectiva de las mujeres y descubrir los protocolos de relaciones con agentes de salud tradicional. Estas técnicas facilitaron además el proceso de triangulación de los datos. Asimismo, durante el proceso de análisis y construcción conceptual se entrevistaron a tres informantes claves: una machi, una peñeñelchefe (matrona o partera tradicional) de las comunidades estudiadas y una kuse (mujer mapuche experta y conocedora de la cultura) en su condición de mujer de 94 años y madre de once hijos; quienes con sus distintas perspectivas contribuyeron a validar las hipótesis emergentes del estudio.

Las observaciones de campo, sesiones grupales y entrevistas fueron transcritas y analizadas utilizando el modelo de teoría fundamentada desarrollada por Strauss y Glaser (1970). Esta teoría plantea generar modelos teóricos a partir de un análisis sistemático de los significados y conceptos que construyen los actores sociales a partir de su experiencia en el mundo cultural. El proceso de saturación y comparación constante de la información fue el enfoque transversal en la construcción teórica de este estudio; lo cual se expresó en el análisis continuo y exhaustivo de las hipótesis conceptuales emergentes de los datos y su consiguiente contrastación empírica en terreno.

Desde el punto de vista analítico el estudio involucró el desarrollo de tres etapas: identificación de temas emergentes, construcción de categorías conceptuales y producción de modelos o cuerpos teóricos. El proceso de análisis de los datos textuales se realizó a través del programa Atlas.ti (Muhr 1997) y consistió en: (1) codificar las narraciones de entrevistas y sesiones grupales, lo cual permitió categorizar, separar y organizar el texto en unidades interpretativas menores; (2) construir familias de códigos (codificación axial) a través de un proceso constante de comparación y saturación de sus significados, y (3) establecer redes de relación entre familias de códigos para descubrir los modelos teóricos que emergen de los datos.

Finalmente, es necesario señalar que el análisis de los datos fue un proceso simultáneo a la recolección y codificación de textos, situación que incidió en la selección de los participantes del estudio y en la búsqueda de informantes claves que permitiesen confirmar o rechazar las teorías emergentes.

\section{Resultados}

Los resultados fueron organizados de acuerdo a cuatro categorías conceptuales que conforman el proceso reproductivo: embarazo, parto, nacimiento y puerperio. En cada uno se describen los temas emergentes y de interés cultural discutidos por las mujeres y se ejemplifica con extractos de sus discursos.

\section{Embarazo: proceso biológico y ético cultural}

El embarazo fue concebido como un acto reproductivo biológico y natural, ajeno a cualquier interpretación mágico-religiosa. El grupo de mujeres entrevistadas planteó que los hijos son siempre bienvenidos, aun si la madre es soltera y se desconoce al progenitor. En este caso, la familia de la embarazada acoge al hijo como un integrante más sin que exista discriminación hacia el niño. No obstante, se consideró que el tener un hijo fuera del matrimonio es un acto que deshonra a la mujer; una de las entrevistadas mencionó: "por aquí pasó eso, la pobre niña andaba en el monte arrancada, tenía vergüenza de llegar a su casa, porque la criaron señorita y con todo eso su familia sufrió mucho, le faltó a su familia”. Por otra parte, el embarazo en adolescentes fue considerado una situación muy delicada, ya que según las mujeres el cuerpo de la niña se encuentra aún en crecimiento; varias de ellas indicaron lo siguiente: "las mujeres de antes se casaban jovencitas, tenían muchos hijos, ahí le entraban enfermedades", "no está bien tener guaguas muy niñitas, los huesos son blandos, no están firmes", "cuando las niñitas dan pecho ahí se le va toda la fuerza y se vuelven enfermizas".

Respecto de cuándo el feto es considerado persona, hubo diferentes opiniones entre las mujeres, algunas indicaron que "desde el comienzo, cuando uno sabe que está embarazada sabe que trae una vida adentro", otras mencionaron que "cuando la guagua se mueve, ya ahí uno piensa que es algo vivo, y que va a ser pues, una persona nomás"; la mayoría de las mujeres aludió a su conocimiento en animales para ejemplificar esta idea, se indicó que "cuando un animalito preñado se muere, ahí se ve la cría casi formada, así mismo le debe pasar a una", "al mes o dos meses es una pelota de sangre como gusano, después ya empieza a parecer gente". En este contexto, el aborto espontáneo fue considerado como la muerte del niño, el que puede ocurrir por una 
trasgresión de la embarazada a las reglas culturales, o por una falta de cuidado de la mujer. Respecto de los abortos provocados, la opinión general fue de desacuerdo con este tipo de procedimientos y mencionaron que no era una práctica realizada por la mujer mapuche, al menos de aquellas que viven en comunidades.

\section{Precauciones durante el embarazo}

Las mujeres revelaron complejos mecanismos de control y prevención biológicos y culturales que operan en la sociedad mapuche para proteger a la embarazada y el niño. Estos consisten en un conjunto de regulaciones que apuntan a preservar la salud de la madre, asegurar la indemnidad del feto y la salud futura del niño. Entre ellas destacan las prohibiciones de consumir algunos alimentos y participar en ciertas actividades sociales y laborales.

\section{Prohibiciones alimentarias y ecológicas}

Respecto de las prohibiciones de alimentación, las mujeres distinguían entre alimentos considerados fríos y calientes. Se indicó que las embarazadas deben evitar el consumo de alimentos pesados, grasosos, con exceso de condimentos, sal, ají, o expuestos largamente al sol, los cuales aumentarían la condición de calidez de su estado. Las siguientes citas ilustran esta idea: "les sube el calor, el ácido y da colitis", "las guaguas salen con su carita roja, con pintas rojas". Asimismo, mencionaron que la embarazada no debería consumir alimentos o líquidos helados, ya que el contraste entre frío y calor produce una enfermedad llamada "pasmo". Entre las descripciones de este fenómeno se encuentran: "se le aprieta la guata", "le da pasmo a la guagua", "se sube la sangre a la cabeza" y "se le produce enfriamiento del estómago". Esta enfermedad se asocia a fenómenos de contracción y dilatación del sistema circulatorio que se expresa en forma inespecífica, ya sea a través de rash cutáneos en diferentes partes del cuerpo: "le entran granos rojos, después se ponen amarillos"; alteraciones del tránsito digestivo y problemas musculares: "dan calambres como tullimiento de las manos o los pies".

Otros alimentos prohibidos para la embarazada son aquellos que provocarían daño o imperfecciones al feto, entre éstos indicaron todos los alimentos gemelares y deformes. Por ejemplo, si la embarazada come huevos de doble yema es probable que tenga hijos gemelos. Durante el debate realizado sobre el embarazo gemelar, las mujeres concluyeron que este tipo de embarazo no es beneficioso para la familia: "no es bueno, trae problemas a los niños, trae desgracia", "siempre hay uno que es débil y se muere", otras mencionaron que: "los antiguos decían que es por un mal, por envidia". El trasfondo de la discusión era la idea de anormalidad de la situación, anormalidad que es explicada a través de la intervención de terceros: personas que tienen la intención de hacer daño a la familia, o castigo del mundo espiritual por alguna falta cometida por la mujer o su familia.

Por otra parte, las mujeres indicaron que durante el embarazo no se pueden ingerir alimentos con algún grado de deformidad, generalmente papas o frutas, ya que esta anomalía es traspasada al feto: "si la mujer come una papa rara así, le sale deforme la guagua, sale enfermo, a veces chuequito".

\section{Observación de fenómenos no naturales}

En relación al tema de la anormalidad en los niños, las mujeres mencionaron que la embarazada no debería experimentar situaciones de gran pena o dolor espiritual, pasar sustos, o tener trafentún (encuentros con espíritus de la cultura mapuche); estas dos últimas situaciones afectan directamente la salud del niño, provocando todo tipo de anormalidades al nacer, como también afectan la salud de la embarazada a quien le quita la energía necesaria para mantener sano al niño que tiene en su vientre, incluso provocándole un aborto espontáneo. Como mecanismos preventivos se recomienda que la embarazada no salga de la casa al caer el sol, dado que puede ver un anchimallen (espíritu con forma de niño o luz brillante), cherrufes (luces destellantes); no debe transitar sola durante el día por lugares apartados en los que pueda encontrarse con un meulen (viento arremolinado provocado por un espíritu), o un wayllepen (espíritu en forma de animal que aparece en lugares desolados, pantanosos; también fue descrito como animal deforme).

Respecto de las prohibiciones de participación en ciertas actividades sociales y culturales, las mujeres mapuche indicaron que la embarazada debe evadir el contacto con personas moribundas o participar de la matanza de animales, ya que observar el sufrimiento o deceso de un ser vivo, sean estos animales o personas, producen el konün (enfermedad en que el aliento del muerto pasa al feto). El konün como 
enfermedad se expresa en forma inespecífica en los niños, desde la deformidad, pasando por episodios frecuentes de enfermedad, hasta alteraciones del carácter o personalidad del niño. Por lo tanto, el efecto del konün puede ser observado hasta algunos años después del nacimiento. $\mathrm{Al}$ respecto, una de las mujeres comentó: "ese niño era flaquito, siempre tenía que estar en el hospital con remedios, lo llevaron a la machi y dijo que era konün, eso era, por eso el niño siempre se enfermaba, no arribaba su salud, era konün, porque ella (la mamá del niño) atendió a su papá hasta que murió, eso era". Para evitar esta enfermedad las mujeres indicaron que el niño debe observar la matanza de un ave o de un animal en sus primeros años de vida.

\section{Actividades laborales}

Otra de las actividades que la embarazada debe evitar es el trabajo fuerte, como cargar elementos pesados o participar en actividades agrícolas que demanden gran esfuerzo; ello provoca koñiwe kutran (caída de la matriz o útero). Según las mujeres esta enfermedad provoca sufrimiento fetal, produce dolor en el parto, sangramiento e incluso el aborto. La explicación que se otorga a este fenómeno es que: "el útero se sale de su lugar, aprieta al niño, que no puede respirar", "la matriz se cae, no está en su sitio". El koñiwe kutran es asociado a su vez al periodo de postparto en el cual la madre debería mantener un reposo relativo, en este caso el útero se suelta y la mujer siente la sensación interna de una masa que se mueve provocando dolor menstrual, cefalea e inflamación del vientre.

\section{Parto y nacimiento: la mujer expuesta a las fuerzas de la naturaleza}

En esta dimensión emergieron dos categorías conceptuales. La primera categoría se refiere a que el evento del parto fue considerado una situación de cuidado con los fluidos corporales, en este caso la sangre y la placenta; la segunda categoría tiene relación con el significado del nacimiento de un niño para la comunidad mapuche.

\section{El parto y los fluidos corporales de la mujer}

Las mujeres entrevistadas mencionaron que el parto constituye un evento de gran fragilidad y riesgo para la mujer mapuche, dado que su condición física es de cuerpo abierto a la naturaleza: "la mujer se abre como un canal, su cuerpo y su espíritu se abren". En esta condición la mujer necesita la protección de sus espíritus, de su familia, de las mujeres más cercanas de su comunidad; por lo tanto, durante el parto ella no puede quedar sola "si ella está solita puede venir un espíritu malo y hacerle daño a ella y al niño", "a veces le toma la cabeza y la vuelve loca, también las pueden hacer sufrir mucho para que salga la guagua". Las mujeres mencionaron además que si no hay cuidado y buen manejo de los fluidos corporales durante el parto, especialmente de la sangre y la placenta, éstas pueden ser utilizadas por personas que le tienen envidia a la familia para producir daño y enfermedad a la mujer o al hijo.

Respecto de la sangre se planteó que todas las cosas que la mujer manche deben ser lavadas en agua corriente, en un arroyo o acequia donde el agua fluya; el agua es concebida además como un elemento purificador del cuerpo. Algunas mujeres mencionaron que: "la gente antigua dice que las mujeres con sus guaguas recién nacidas se bañaban en el río, pero eso ya no se hace más, ahora todas vamos al hospital y no se pueden llevar esas costumbres". Otras mujeres dijeron que la sangre también puede ser quemada en el fogón, "no deben quedar trapos con sangre por ahí, le pueden hacer mal a la mujer con eso".

Respecto de la placenta las mujeres mencionaron que ésta se lee, interpreta y dispone de ella de acuerdo a las reglas de la cultura mapuche. La lectura de la placenta fue concebida como un acto cultural vital para conocer el destino del niño y evitarle sufrimientos en la vida. Esta lectura es realizada por mujeres expertas de la comunidad, quienes ayudan a la parturienta a tener sus hijos: "la peñeñelchefe (partera, matrona empírica) mira bien la placenta, la da vuelta con cuidado y le dice todo lo que le va a pasar, dice cómo va a ser el niño, cómo va a ser su carácter, así uno ya está preparada". Por otra parte, se planteó que la placenta tiene pertenencia, pues lleva el espíritu de la madre y del niño, por lo tanto debe tener una buena disposición final. Las mujeres mencionaron que la placenta debe ser enterrada debajo de un árbol nativo o frutal, lo cual daría protección y fortaleza física al niño.

De las mujeres entrevistadas, cuatro habían tenido sus hijos mayores en la casa y ayudadas por su suegra, un familiar cercano, o una partera que llegaba al menos un día antes del nacimiento del 
niño, especialmente si la madre era primigesta, o el hijo no se encontraba en posición cefálica-caudal, en este caso la partera realizaba masajes en el vientre para acomodar al niño. Algunas mujeres mencionaron que acudían a la partera durante el embarazo para que le acomodara la guagua, la que a veces se atravesaba o incrustaba en el abdomen producto del trabajo excesivo; una de las mujeres relató lo siguiente: "yo me la pasaba lavando en la artesa, a veces con harto frío y me mojaba la guata, mi guagua parece que sentía ese frío y se apretaba, yo iba donde la sra. M. y me sobaba suavecito con hojitas de maqui, cuando se soban le sale un aceitito, ahí quedaba bien de nuevo, yo fui como cuatro veces".

La partera acompañaba y preparaba a la futura madre para tener un buen parto, el que consistía en un trabajo sin dolor excesivo, con la placenta expulsada inmediatamente después del nacimiento y dejando a la madre con una buena técnica de amamantamiento. Todos los relatos coincidían en que el parto fue en posición hincada, al lado de la cama, sobre una sábana limpia y jalando de un lazo o una manta que colgaba del techo. Las mujeres quienes no habían tenido esa experiencia contaban que sus madres o abuelas relataban la misma situación: "las mujeres se achulluncaban, la matrona la apretaba por la espalda y cuando estaba lista recibía la guagua, así se sufría menos y era mas rápido", "la matrona le apretaba el estómago para que salga la placenta, le sobaba, si no salía la ayudaba con la mano", "le daba lawén (hierbas medicinales) en tomas calientes, para que suelte la sangre que queda apelotonada, así soltaba restos de la placenta para que no le quede adentro".

Dado que el embarazo y parto son de acuerdo a la lógica médica cultural, estados cálidos, la mujer debe evitar exponerse a corrientes de aire frío. En consecuencia, el ambiente en donde se realice la atención del parto debe estar tibio y protegido de corrientes de aire. Respecto del corte y cuidado del cordón umbilical, la partera informante de este estudio mencionó lo siguiente: "lo corto con una tijera o un cuchillo filoso limpio, lo amarro con una pitilla de saco, le coloco jugo de matico y lo tapo con una fajita de género limpio". La mayoría de las mujeres mencionó que fajaban a los recién nacidos hasta que el cordón umbilical se secaba y caía solo; relataban que la indicación en el hospital era de acudir al centro de salud más cercano para que la enfermera cure la zona, sin embargo la mayoría declaró no seguir esta práctica por problemas de acceso a la posta. Por ejemplo, una mujer dijo:

yo no voy porque la posta queda lejos, hay que sacar a la guagua al viento y se puede enfermar, yo lo hago como lo hacía mi mamá, le pongo jugo de matico y un trapito con una fajita, antes hacían fajas especiales para los niños, pero ahora ya no hay esa costumbre, yo le pongo una faja de género que le apriete la guatita para que no se le infle esa pelota cuando llora.

Algunas mujeres dijeron que guardaban el cordón de sus hijos porque "trae buena suerte", otras dijeron que "antes se ponía en un saquito de lana y se colgaba al lado del fogón, para que el niño esté siempre bien, pero ahora ya no, a veces se cae sin que uno se dé cuenta y se va con el pañal".

El cuidado inmediato de la madre al finalizar el trabajo de parto consiste en abrigarla, alimentarla con una sopa caliente de ave y reposo en cama con su hijo recién nacido. Algunas mujeres indicaron que durante dos o tres días la puérpera bebía en la mañana una taza de vino tinto con harina tostada (chupilca) para reponer la sangre. El relato de esta experiencia dice: "la mamá toma una chupilca por la mañana, dicen que el vino tinto ayuda a reponer la sangre que se perdió, la sangre se pone más delgada y el vino tinto ayuda a la sangre".

La mayoría de las mujeres dijeron que el parto hospitalario fue introducido por los médicos con el propósito de proteger a la embarazada y a su hijo de enfermedades o de la muerte. Ninguna indicó que esta situación fuese negativa para la mujer, no obstante se plantearon una serie de objeciones al proceso de atención en el hospital, entre éstos, el uso de instrumental obstétrico, la revisión del canal de parto cada vez que examinan a la madre, la soledad en que se realiza el procedimiento, la falta de cuidados con la sangre y la placenta, la comida en exceso liviana y el baño que se exige antes o inmediatamente después del parto. Además de estas inquietudes las mujeres mencionaron que les preocupaba ausentarse del hogar, dejar los niños, los animales, la huerta, la chacra. Los siguientes extractos sintetizan estas ideas: "me tuvieron dos días en el hospital, me decían que todavía no estaba lista pero que no me podía volver, me miraban el interior a cada rato, tuve mi guagua ahí sola, no había nadie (de la familia) porque no hay plata 
para viajar", "pasé mucha hambre porque dan puras sopitas y gelatina", "yo vi que un hombre andaba trapeando la sangre de la sala, las cosas caen a un balde que junta todo y después será que lo votan por ahí como cualquier cosa".

\section{El nacimiento como evento cultural de refor- zamiento de la identidad étnica}

El nacimiento de un niño fue considerado un acontecimiento de gran importancia social y cultural no tan sólo para la familia, sino también para la comunidad. Para ésta, el nacimiento representa la perdurabilidad de la sociedad mapuche, es la forma en que el pueblo permanece y se reproduce históricamente: "un niño o niña siempre es bienvenido, otro mapuche para que no se termine nuestro pueblo, para que siga adelante". El nacimiento de un niño refuerza la identidad cultural del grupo a través de una de las ceremonias más relevantes en este contexto, la asignación del nombre del niño, el lakutún. Las mujeres señalaron que esta actividad era muy importante entre los mapuche antiguos pero que se está perdiendo en las comunidades, "antes se hacía una fiesta, se mataba chancho o cordero, venía el abuelo porque el niño llevaba su nombre, así tenía que ser, pero ahora se le coloca cualquier nombre que le guste a uno".

Los cuidados del recién nacido involucran fundamentalmente mantener la fontanela abrigada; una de las mujeres ejemplificó el cuidado de esta zona diciendo: "eso está abierto, puede tomar frío y enfermar a la guagua, se le pone gorrito de lana hasta que se cierra; como a los tres meses ya está más cerrado, antes le ponían una motita de lana y el gorrito, pero con el gorrito está bien". Las mujeres mayores señalaron que fajaban a sus hijos desde debajo de los brazos hasta los pies, para que crezcan derechos, no obstante muchas dijeron que esa práctica no se continuaba porque ahora se sabía que podía perjudicar al niño.

\section{En el puerperio o posparto confluyen ideas de la cultura popular}

El puerperio fue considerado un período de fragilidad para la salud de la madre, la que debe mantenerse en cuarentena. Esto significa que durante cuarenta días la madre debe evitar cambios bruscos de temperatura, exponerse largamente al sol y evitar trabajos pesados. Al respecto las madres relataron: "no se debe asolear la cabeza cuando anda en la huerta", "se tiene que abrigar la espalda para salir al aire o dar pecho", "la matriz está suelta, todavía le queda sangre pegada, si hace fuerza se le cae la matriz, a veces vuelve a sangrar". Las mujeres mencionaron que antiguamente los mapuche se bañaban en un estero de agua corriente inmediatamente después del parto, pero que esta práctica se ha perdido por temor al sobreparto, por lo tanto la indicación actual es: "lavarse todos los días los interiores con agua de matico tibia y evitar los baños de agua fría". El sobreparto (domo kutran o pura molfun) es una enfermedad que produce mucha aprensión entre las mujeres; se manifiesta con dolores de cabeza, espalda, sangramientos o dolores menstruales, los que pueden aparecer hasta años después de producido el parto. Asimismo, se mencionó que durante la cuarentena la mujer debe evitar las relaciones sexuales, al menos hasta que su cuerpo se encuentre fortalecido, algunas mencionaron un mes de abstinencia sexual y otras 40 días. Muchas mujeres señalaron que amamantar es una forma de prevenir los embarazos, aunque algunas indicaron que posterior a la cuarentena comienzan a utilizar métodos anticonceptivos basados en hierbas medicinales. Respecto al amamantamiento, todas las mujeres dijeron que el calostro es la leche más nutritiva de la madre "es la que tiene más alimento, y hace que el niño sea más sanito", la limpieza del pezón se realiza con una infusión de matico.

En el contexto de la cosmovisión mapuche de enfermedad las mujeres no plantearon cuidados específicos para la madre. A partir del nacimiento, la mujer comienza a mantener las reglas de la cultura como cualquier otro miembro de la sociedad mapuche.

\section{Discusión y Conclusiones}

Los resultados demuestran que en el proceso de reproducción humana mapuche, tanto el modelo de salud tradicional indígena y popular, así como las normas sociales y culturales de la sociedad mapuche moldean la percepción y creencias que las mujeres tienen respecto del embarazo, parto y puerperio. Los modelos teóricos emergentes del análisis de los datos apuntan hacia dos elementos sobre los cuales se construyen los patrones reproductivos en la sociedad mapuche; primero "la existencia de un modelo etnofisiológico capaz de armonizar explicaciones biológicas y culturales", y segundo 
"el proceso reproductivo de la sociedad mapuche actual está moldeado por las estructuras sociales y políticas del Estado chileno". Estos hallazgos son coherentes con los planteamientos generales de la antropología de la reproducción, en que se postula la presencia de conceptos de fisiología basados en una epistemología propia del conocimiento indígena que dirige las prácticas rituales, terapéuticas y reproductivas (McClain 1989; Newman 1995), y la presencia de estructuras sociales y políticas que presionan y moldean las decisiones reproductivas de la población, como por ejemplo biomedicación del parto, pobreza, migración (Goforth 1988; Laderman 1997).

Los eventos reproductivos descritos tienen una connotación absolutamente natural para la vida de la mujer. El embarazo fue concebido como producto de una relación sexual entre un hombre y una mujer, en el que se excluyen explicaciones sobrenaturales tales como poblamiento del cuerpo femenino por seres extraños, o contactos indirectos, como es el caso en otras culturas de Chile y América Latina. Durante este período la mujer mantiene su ritmo de trabajo habitual, el que sólo disminuye en esfuerzo al acercarse la fecha del parto, ello tanto por razones propias de su condición física, como para evitar un parto complicado. Factores económicos están además presentes en esta apreciación, ya que la mujer es parte fundamental de la fuerza laboral y productiva de la familia campesina.

Aun cuando el embarazo es una condición que no afecta profundamente las labores cotidianas de la mujer, existe un modelo de cuidado cultural específico para la embarazada que denota su grado de fragilidad física y espiritual. Un conjunto de normas sociales y culturales definen lo que es y no es permitido para la embarazada mapuche, entre las que destacan reglas rigurosas de alimentación, prohibición de participar en algunos eventos y prevenir el encuentro con ciertos espíritus o seres de la cultura mapuche que puedan dañar a la embarazada o al feto. Por otra parte, en los cuidados sugeridos durante el embarazo, parto y puerperio se manifiesta un importante componente conceptual de la medicina popular, cuyo paradigma se basa en la oposición de frío y calor y la mantención de un equilibrio entre estos dos estados. El embarazo concebido como estado cálido gatilla complejos mecanismos de mantención del equilibrio corporal, particularmente asociados a la alimentación y mantención de la temperatura, por ejemplo, evitar exponerse al frío o calor extremo, a corrientes de aire, ingerir alimentos en exceso fríos. Diversos estudios en antropología médica han demostrado que estas precauciones se encuentran ampliamente distribuidas en mujeres de culturas populares urbanas de Latinoamérica (Foster 1998; Scheper-Hughes 1992).

Por otra parte, la concepción mapuche de salud y enfermedad se manifiesta en la presencia de seres, fenómenos naturales y espíritus que pueblan el territorio mapuche y cuyos encuentros con la embarazada le provocan problemas físicos o espirituales; de aquí que la embarazada evite circular sola y por espacios donde habite un meulen, wayllepen, anchimalen, cherrufe, entre otros. Del mismo modo, el contacto con moribundos, presenciar sufrimiento, ingerir $\mathrm{u}$ observar alimentos gemelares o deformes inducen a enfermedades de la embarazada y malformaciones del feto. Antecedentes de este tipo son descritos en estudios realizados por Gusinde (1917) y Oyarce (1988), lo cual advierte sobre la perdurabilidad de ciertos contenidos culturales relacionados con la cosmovisión del mundo y la naturaleza mapuche.

Respecto del parto, se aprecia un importante cambio en las prácticas culturales mapuche dado naturalmente por las altas tasas de profesionalización de la atención a la embarazada. A pesar de que el parto domiciliario se ha suprimido totalmente en las comunidades de este estudio, en el discurso de las mujeres aún está presente el contenido de los relatos de madres y abuelas quienes tenían sus hijos en casa, ayudadas por matronas empíricas. Al respecto, mencionan la pérdida de al menos cuatro elementos culturales considerados vitales para la embarazada y su hijo(a): el acompañamiento y apoyo de mujeres experimentadas durante el parto, la lectura y disposición de la placenta, el cuidado con los fluidos corporales y la alimentación tradicional para recuperar las fuerzas de la mujer.

El puerperio es una etapa que se desarrolla en el espacio de la familia y la comunidad. En ella retornan las prácticas culturales mapuche en el cuidado de la madre y el recién nacido. Las percepciones y creencias acerca de este proceso están, al igual que en el embarazo, moldeadas por concepciones de la medicina mapuche y popular. La noción de equilibrio entre los elementos fríos y cálidos prevalece como un factor que lidera la práctica del cuidado postnatal: corrientes de aire, cambios bruscos de temperatura, exposición prolongada de la cabeza al sol, son factores de riesgo ambiental que producen alteraciones de la 
lactancia (corte de la leche) y enfermedades a la madre. Este período de fragilidad y cuidado es denominado cuarentena. En éste la mujer además de mantener las precauciones antes mencionadas, debe evitar las relaciones sexuales, cargar objetos pesados y realizar todo tipo de esfuerzo físico. La cuarentena ha sido un síndrome cultural ampliamente observado en la cultura popular chilena y latinoamericana (Citarella et al. 2000; Modena 2002). Respecto de los cuidados de la mujer y el niño, no se observaron precauciones o normativas desde la cosmovisión mapuche debido a su condición particular de puérpera. En consecuencia el cuidado y prevención de enfermedades se rige por el cumplimiento de las normas culturales y sociales de cualquier persona mapuche.

Considerando los antecedentes aportados por trabajos historiográficos de las décadas de 1940 a 1960, los resultados de este estudio dan cuenta de un importante proceso de cambio en la cultura mapuche respecto del proceso de reproducción biológica y cultural. En los relatos de las mujeres estuvo siempre presente un discurso sobre el antes y el después, de lo que hacían y creían las abuelas o antiguas y lo que hoy se piensa y practica. Aquello demuestra la dinámica de los fenómenos culturales cuando los sujetos transitan de un espacio social y cultural a otro, o cuando son impelidos forzosamente a cumplir los patrones de una cultura hegemónica. Las mujeres, en su condición de embarazada o puérpera, han reelaborado una serie de significaciones sobre estos procesos, los cuales desde un punto de vista estrictamente conceptual se acercan más al modelo naturalístico de la medicina popular chilena que al de la medicina tradicional mapuche.

En resumen, el estudio confirma que la reproducción humana no es un fenómeno exclusivamente biológico, que la sociedad mapuche tiene una serie de patrones culturales y sociales para el cuidado y protección del embarazo, parto y puerperio, los que en último término implican un importante resguardo de la vida humana. El conocer estas prácticas y percepciones contribuye a generar modelos de atención de salud culturalmente pertinentes para la mujer y su familia.

Agradecimientos: Especial gratitud nos merecen las mujeres quienes depositaron su confianza en el equipo investigador para contar sus relatos e historias de vida (Sonia L., Soledad M., Luisa M., Elena M., Yolanda S., Jovita Z., Elvira C., Sonia LL., Teolinda C., Isabel H., Rosa A. y Nidia B.). A los Sres. Aldo Vidal y Wilfred Diener por corregir este escrito. Agradecemos los comentarios de los evaluadores, los cuales contribuyeron sustantivamente a mejorar la calidad de este manuscrito.

\section{Referencias Citadas}

Alarcón, A.M., A.Vidal y J. Neira

2004 Política de salud intercultural: perspectiva de usuarios mapuche y equipos de salud de la IX Región. Revista Médica de Chile 132:1109-1114.

Andrade, R.

1992 Medicina en la Araucanía 1850-1900. Tesis para optar al grado de Licenciado en Educación, Departamento de Humanidades, Universidad de La Frontera, Temuco.

Beneria, L. y M. Roldán

1987 The Crossroads of Class and Gender. University of Chicago Press, Chicago.

Browner, C. y C. Sargent

1996 Anthropology and studies of human reproduction. En Medical Anthropology Contemporary Theory and Methods, editado por C. Sargent y T. Johnson, pp. 183-219, Praeger, CT.

Citarella, L., A.M. Conejeros, B. Espinosa, I. Jelves, A.M. Oyarce y A.Vidal

2000 Medicinas y Culturas en la Araucanía. Segunda edición. Editorial Sudamericana, Santiago.

Faron, L.

1964 Hawks of the Sun. Mapuche Morality and its Ritual Attributes. University of Pittsburgh Press, Pittsburgh.
Foster, G.

1998 Diseases etiologies in non-western societies. En Understanding and Applying Medical Anthropology, editado por P. Brown, pp. 108-118. Mayfield Publishing Company, California.

García Canclini, N.

2005 Culturas Híbridas. Estrategias para entrar y salir de la Modernidad. Paidós, Barcelona.

Goforth, L.

1988 Household Structure and Birth Attendant Choice in a Yucatec Maya Community. University of California, Los Ángeles.

Guevara, T.

1912 Las últimas familias i costumbres Araucanas. Anales de la Universidad de Chile 80:215-342.

Gusinde, M.

1917 Medicina e higiene entre los Araucanos. Revista Chilena de Historia y Geografía 22:382-415.

Harris O. y K. Young

1991 Engendered Structures: Some Problems in the Analysis of Reproduction. Macmillan, London.

Hillger, I.

1957 Araucanian Child Life and its Cultural Background. Smithsonian Institute, Washington. 
Laderman, C.

1997 Wives and Midwives: Childbirth and Nutrition. University of California Press, Berkeley.

McClain, C.

1989 Women as Healers. New Brunswick Rutgers University Press, New Jersey.

Modena, M.E.

2002 Combinar recursos curativos: un pueblo mexicano en las últimas décadas del siglo XX. En Entre Médicos y Curanderos, editado por D. Armus, pp. 333-371. Grupo Editorial Norma, Buenos Aires.

Muhr, T.

1997 Atlas.ti: Scientific Software Development. Berlin.

Newman, L.

1995 Women's Medicine. New Brunswick Rutgers University Press, New Jersey.
Oyarce, A.M.

1988 La salud entre los Mapuches. Revista Experiencias $3: 1-44$.

Scheper-Hughes, N.

1992 Death Without Weeping: The Violence of Everyday Life in Brazil. University of California Press, California.

Stephen, L.

1991 Zapotec Women. University of Texas Press, Austin. Strauss, A. y B. Glaser

1970 Discovery of Substantive Theory: A Basic Strategy Underlying Qualitative Research. Markham, Chicago.

Wolf, E.

1999 Envisioning Power. Ideologies of Dominance and Crisis. University of California Press, California. 